\title{
Das Ausbreitungsklima der Innerschweiz
}

\begin{abstract}
Summary
The aim of this study is to determine the spatiotemporal structure of the dispersion climate over Central Switzerland. For that reason the investigations have been divided into two parts. The first part deals with the analysis of the temperature stratification which is an important indicator of the vertical diffusion. It was carried out by using IR satellite data as well as temperature measurements and fog observations. The second part is concerned with a detailed analysis of the wind field. Measurements on more than 50 stations have been temporally interpolated and compared with numerical simulations.
\end{abstract}

\section{Einleitung}

Unter dem Begriff Ausbreitungsklima soll im folgenden die Gesamtheitjener klimatologisch relevanten Prozesse verstanden werden, die den Transport und die Verdünnung von Luftschadstoffen beeinflussen. Es sind dies in erster Linie das Windfeld und das Turbulenzverhalten, die zusammen mit den ablaufenden Umwandlungsprozessen als Transmission bezeichnet werden. Mit Hilfe von Computermodellen können nach Eingabe von Emissions- und Ausbreitungsangaben Immissionsrechnungen durchgeführt werden.

Das Ausbreitungsklima der Innerschweiz wurde von einer Gruppe des Geographischen Instituts der Universität Bern (GIUB) im Auftrag der sechs Innerschweizer Kantone flächendeckend untersucht. In einem zusammenfassenden Überblick werden die wichtigsten Resultate und die angewandten Methoden der Studie präsentiert. In Figur 1 sind schematisch die Arbeitsgänge dargestellt. Es wird deutlich der Versuch sichtbar, aus Informationen zur Vertikaltemperaturschichtung und zum Horizontalwindfeld geeignete Ausbreitungsstatistiken abzuleiten.

\section{Vertikaltemperaturschichtung, Kaltluftverteilung und Turbulenzverhalten}

Das allgemeine Turbulenzverhalten wird, abgesehen von seiner Abhängigkeit von synoptischen Prozessen, stark durch die oberflächeninduzierte, mechanisch und thermisch bedingte Turbulenzproduktion beeinflußt. Diese Turbulenz ist vor allem dann minimal, wenn weite Teile des Untersuchungsgebietes von stabilen Kaltluftschichten bedeckt werden. Ein erster Arbeitsschritt galt folglich der Erfassung der raumzeitlichen Struktur von Kaltluftverteilung und Temperaturschichtung.

\subsection{Methodik}

Für das Gebiet der Innerschweiz standen mehrere NOAA-Satelliten-Aufnahmen im IR-Spektralbereich mit einer Auflösung von zirka $1 \mathrm{~km}^{2}$ zur Verfügung. Die Temperaturen wurden über bestimmte Höhenintervalle gemittelt. In einem zweiten Schritt wurden die Abweichungen der einzelnen Pixel-Temperaturen von diesen Intervall-Mittelwerten berechnet. Schließlich wurden alle Bilder digital überlagert. Daraus konnte eine Mittelkarte der Oberflächentemperaturen für Strahlungsnächte hergestellt werden (Fig. 2). Aufgrund der Annahme, daß sich tiefe Bodenoberflächentemperaturen bei austauscharmen Wetterlagen auch auf die bodennächste Luftschicht «übertragen», konnten dann Gebiete mit häufigen Kaltluftansammlungen während Strahlungsnächten direkt identifiziert werden. Ein Vergleich zwischen der Größe der Gebiete und der Topographie ließ zudem auch vorläufige, wegen der relativ kleinen Auflösung aber erst grobe Rückschlüsse auf die Mächtigkeit der Kaltluftseen zu.

Fragen nach der raumzeitlichen Dynamik von Kaltluftseen im regionalen Maßstab müssen weiterhin mit traditionellen Verfahren abgeklärt werden. Hierzu standen einerseits Sondierdaten von Payerne und Merenschwand zur Verfügung (je zwei vertikale Meßreihen pro Tag), anderseits die Daten von Temperaturstationen auf vier unterschiedlichen Höhenniveaus im Raum Luzern. Die Auswertungen geben neben Häufigkeitsinformationen auch einen Überblick über die zeitliche Dynamik des Inversionsaufbaus und -abbaus.

Wie schon oben erwähnt, ist der Bodennebel ein guter Indikator für Bodeninversionen und Kaltluftansammlungen. Die Auswertung der Nebelbeobachtungen der SMA an über 40 Stationen im Raum Innerschweiz lieferte weitere Informationen über die Verbreitung und die Häufigkeit von Kaltluftansammlungen in den einzelnen Gebieten.

Beat Rickli, Lic. phil. nat., Geograph, und Heinz Wanner, Prof. Dr. phil. nat., Geographisches Institut, Hallerstraße 12, $\mathrm{CH}$ 3012 Bern, Paul Filliger, Dr. phil. nat., Bundesamt für Umwelt, Wald und Landschaft, Laupenstraße 4, CH-3003 Bern. 


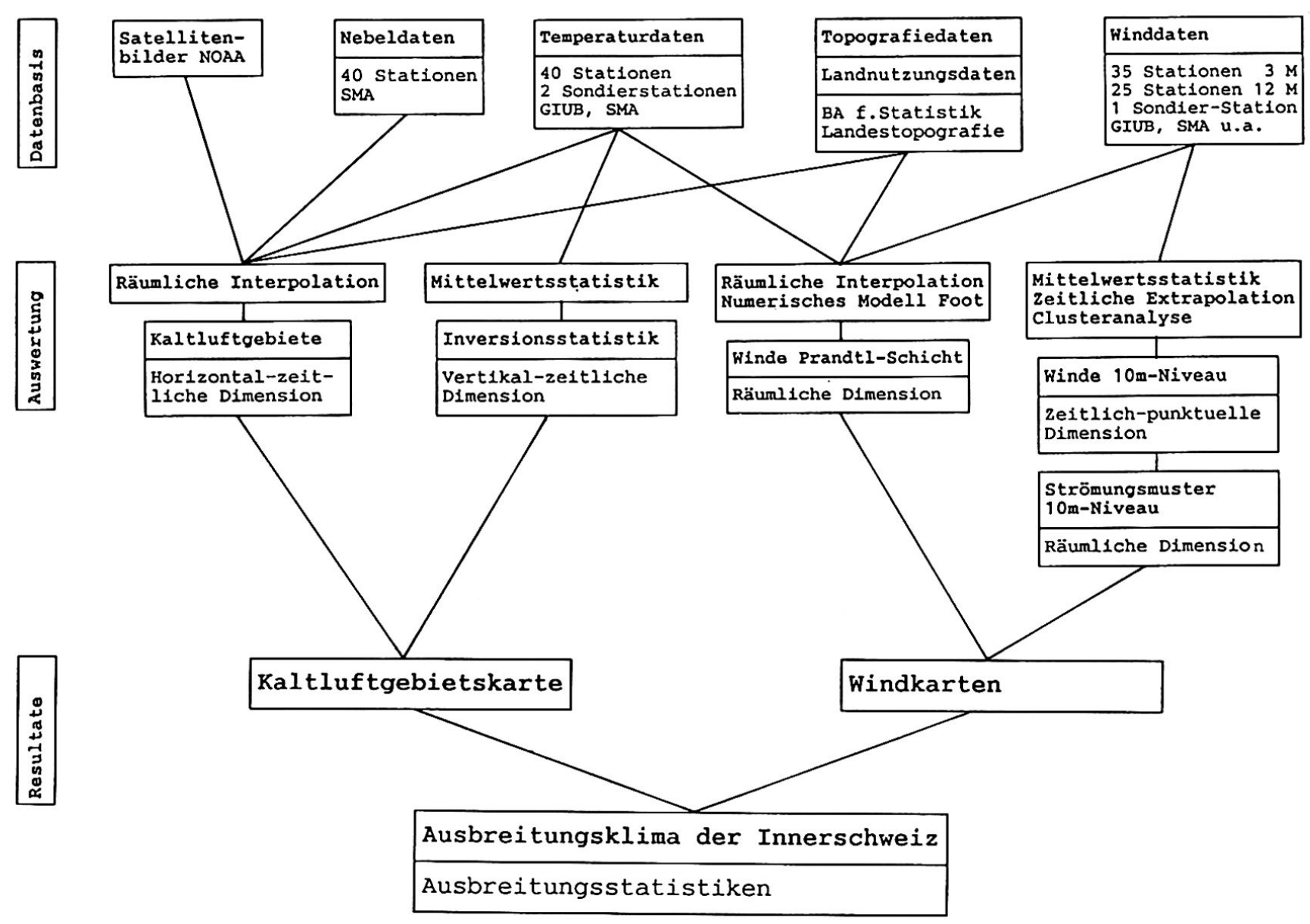

Fig.1 Arbeitsschema zur Studie über das Ausbreitungsklima der Innerschweiz.

Da aber auch Bodeninversionen ohne Nebel auftreten, können mit den Nebeldaten alleine nicht alle Kaltluftgebiete erfaßt werden. Deshalb konnte erst nach einer Synthese aller bisherigen Auswertungen (Satellitenbilder, topographische Angaben, Temperatur- und Nebeldaten) die geplante Kaltluftgebietskarte erstellt werden.

\subsection{Resultate}

Die Bodenoberflächentemperaturen während Strahlungsnächten sind in Figur 2 dargestellt. Interessant ist, daß nicht allein Muldenlagen und auch nicht alle Muldenlagen tiefe Temperaturen aufweisen, was teilweise auf die unterschiedliche Bodenbeschaffenheit und -bedeckung zurückzuführen ist. So weist z. B. die Hochmoorregion von Rothenthurm bei allen Thermalbildern die Intensitätsstufe «sehr kalt» auf.

In $60-70 \%$ aller Sommernächte treten Bodeninversionen mit Kaltluftmächtigkeiten unterhalb von $240 \mathrm{~m}$ auf, die sich am Morgen relativ schnell auflösen (Fig. 3). Im Winter sind Bodeninversionen seltener als im Sommer, ihre
Auflösung erfolgt - wenn überhaupt - erst im Verlauf des Tages.

Inversionsbasen im Bereich zwischen 240 und $950 \mathrm{~m}$ ü.G. finden sich vor allem im Herbst und im Winter im Mittel durchschnittlich an jedem zweiten Tag (mehr als $50 \%$, im Sommer dagegen nur zirka an jedem fünften Tag (weniger als 20\%).

Bei der Interpretation der Nebeldaten fallen die großen Unterschiede zwischen den einzelnen Stationen auf: Alpthal, Bisisthal und Luthern weisen beispielsweise im Winter weniger als fünf Nebeltage auf, andere Stationen, wie Oberiberg, Stans oder Andermatt, mehr als deren 60 (vgl. Beitrag von TROXLER und WANNER in diesem Heft). Als Nebeltage gelten Tage, an denen morgens um 07.00 Uhr die Sichtweite geringer als $1000 \mathrm{~m}$ ist.

Die Kaltluftgebietskarte entspricht der Synthese aller bisher beschriebenen Auswertungen. Sie unterscheidet fünf Klassen, die implizit auch je einem lufihygienischen $\mathrm{Ge}$ fährdungspotential entsprechen: Die Ausbreitungsbedingungen sind dort besonders schlecht, wo die Inversionen häufig tief liegen und gut ausgeprägt sind. Der vertikale Ausbreitungsraum ist hier stark eingeschränkt. Während 


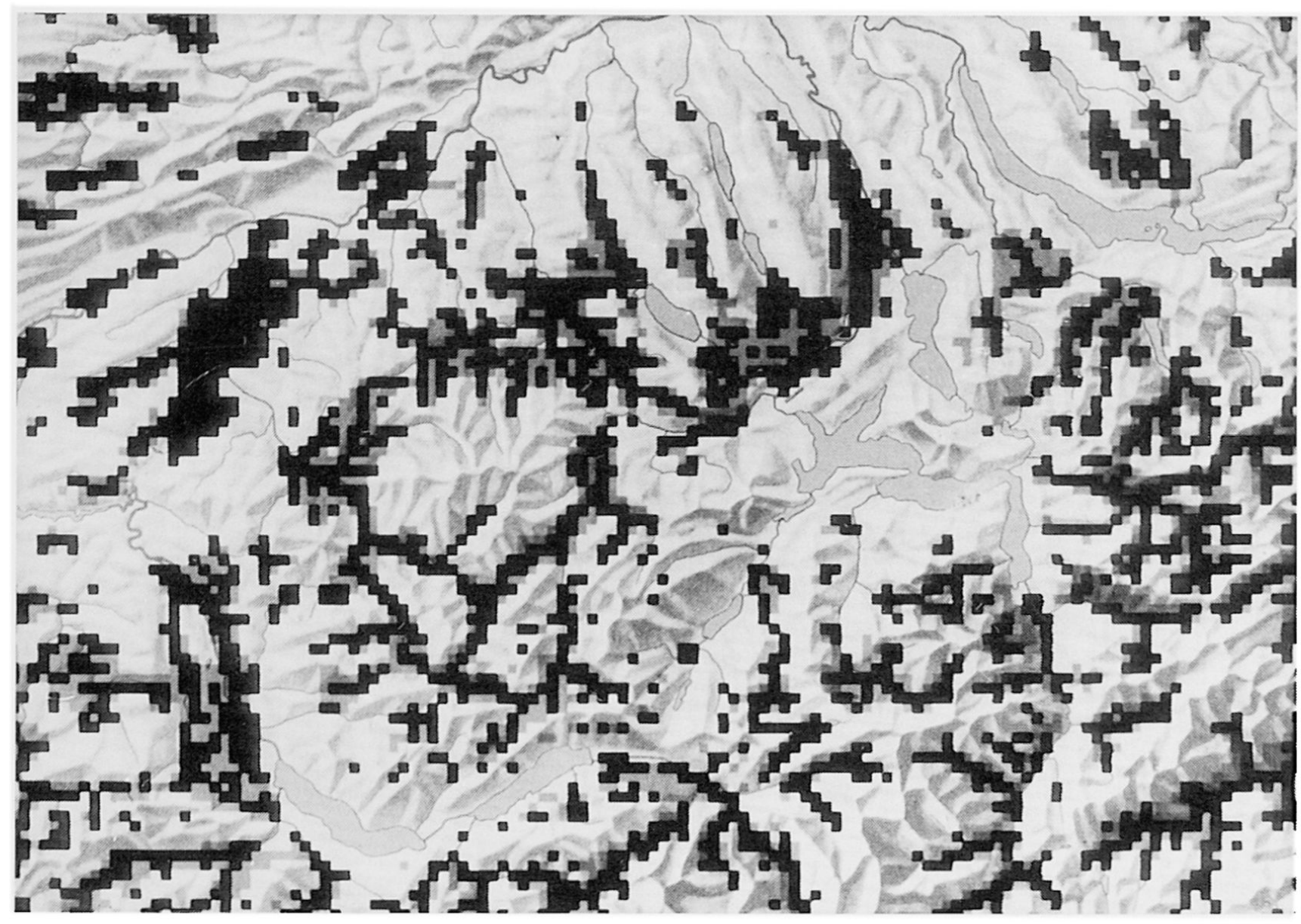

Fig.2 Räumliche Verteilung der Bodenoberflächentemperaturen während Strahlungsnächten, bestimmt aus Thermalbildern des NOAA-Satelliten (Auswertung: G. NEJEDLY). Reproduziert mit Bewilligung des Bundesamtes für Landestopographie vom 19.12.1990.

derartigen Inversionsphasen muß in diesen Gebieten zudem auch mit sehr kleinen Windgeschwindigkeiten gerechnet werden, da die bodennahen Winde von den Höhenwinden entkoppelt sind, so daß auch die Ausbreitung in horizontaler Richtung nur langsam vor sich geht. Es fällt auf, daß die am dichtesten besiedelten Zonen, wie der größte Teil der Agglomeration Luzern oder auch die Städte Cham und Zug, in diesen lufthygienisch ungünstigen Gebieten liegen (Fig.4).

\section{Windfeld}

Horizontale Transporte von Luftschadstoffen werden durch den Wind verursacht, der die Geschwindigkeit und die Richtung des Transports definiert, dabei via die Rauhigkeit (Bodenbedeckung) und Oberflächenerwärmung mechanische und thermische Turbulenzenergie aufnimmt und damit die Verdünnung von Schadstoffen verstärkt. Bodennahe Winde weisen deshalb oft einen mittleren Tages- und Jahresgang auf. Damit eine räumliche und zeitliche Charakterisierung der Windverhältnisse möglich wird, müssen demnach für möglichst viele Stationen Winddaten für mindestens je ein Jahr vorliegen.

\subsection{Methodik}

Die Windverhältnisse in der Innerschweiz wurden an 35 ausgewählten Standorten während vier Phasen zu je drei Monaten gemessen. Anschließend wurde eine statistische Methode entwickelt, die eine zeitliche Extrapolation der vierteljährlichen Meßreihen auf ein Jahr erlaubte, dies vor allem mit Hilfe der im Gebiet permanent messenden Windstationen der SMA. Die relativ aufwendige statistische Extrapolation beruht auf einer Mischung zwischen Clusteranalyse (Schätzung der Windrichtung) und Regressionsanalyse (Schätzung der Windstärken): Für jeden an einer Station A zu generierenden Windmittelwert ist das momentane Strömungsmuster der umliegenden permanent messenden Stationen mit allen Strömungsmustern für die Phase mit Messungen an der Station A verglichen worden und die zur Zeit des ähnlichsten Strömungsmusters herrschende Windrichtung an der Station A als Extrapolationswert übernommen wor- 


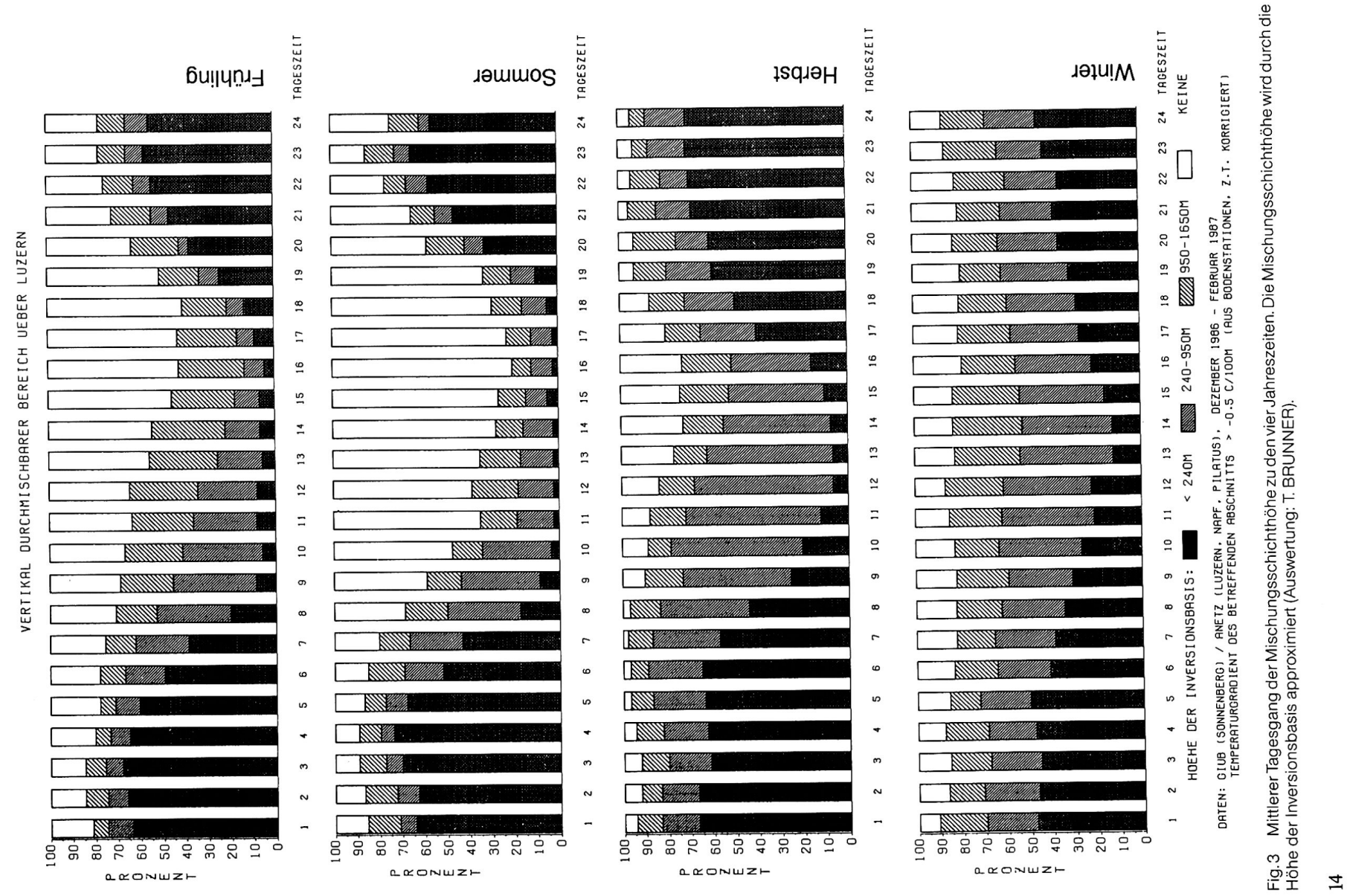




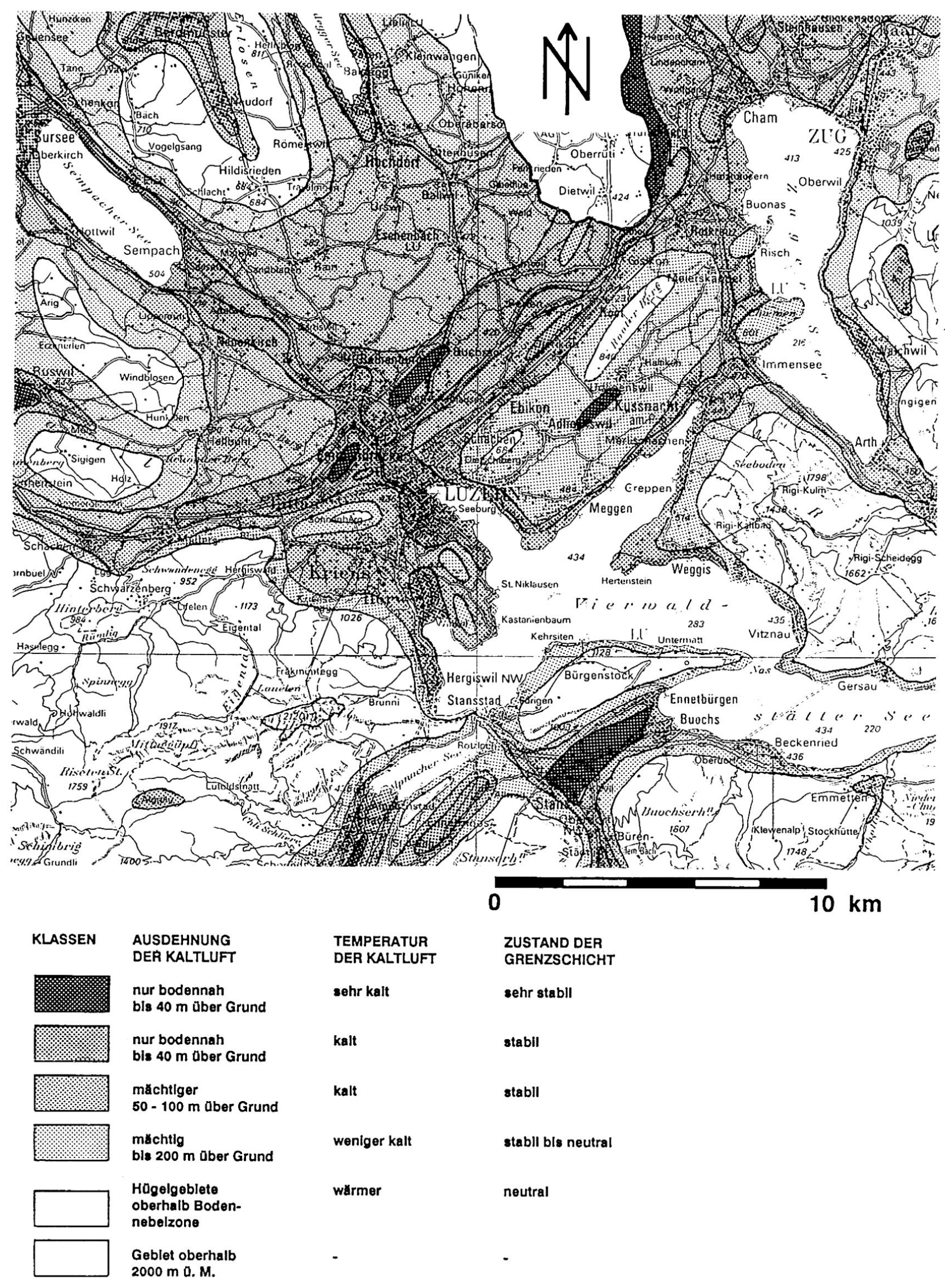

Fig. 4 Modifizierter Ausschnitt aus der farbigen Kaltluftgebietskarte der Innerschweiz (Auswertung: F.X. TROXLER). Reproduziert mit Bewilligung des Bundesamtes für Landestopographie vom 19.12.1990. 


\section{Messung Regressionsmodell Euklidmodell Mischmodell}

\section{Haeufigkeitsverteilung der Winde (3, 6 und $9 \%$-Kreisskalen, 10 Grad-Sektoren)}
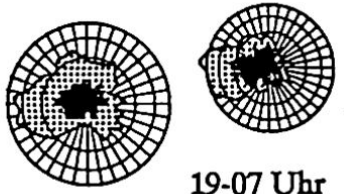

19-07 Uhr

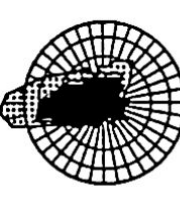

24 Stunden

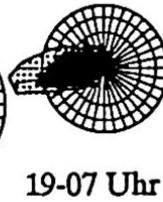

19-07 Uhr

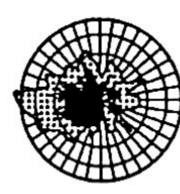

24 Stunden

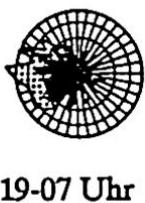

19-07 Uhr

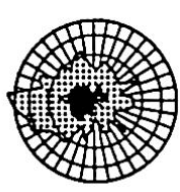

24 Stunden

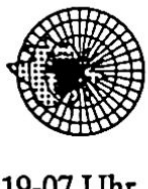

19-07 Uhr

24 Stunden
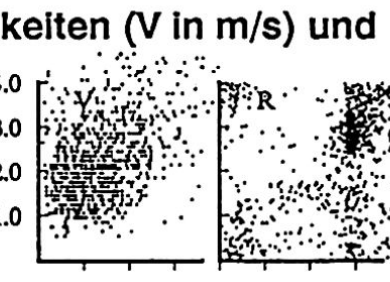

1.02 .03 .0

Windrichtungen ( $R$ in Grad)
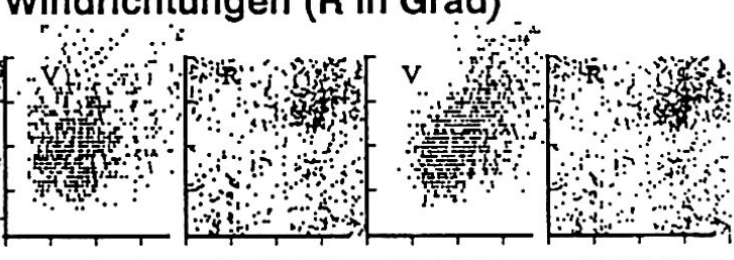

Nordkomponenten ( $\mathrm{N}$ in $\mathrm{m} / \mathrm{s}$ ), E-Komponenten ( $\mathrm{E}$ in $\mathrm{m} / \mathrm{s}$ )

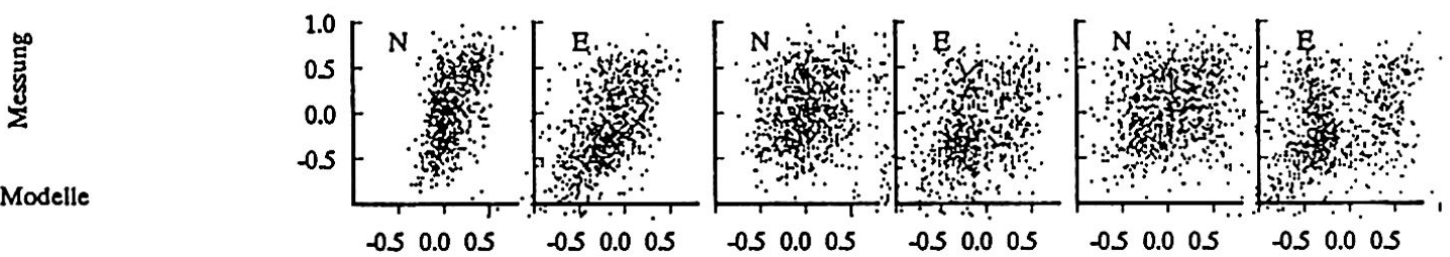

Mittlerer Tagesgang der Windgeschwindigkeiten (in $\mathrm{m} / \mathrm{s}$ )

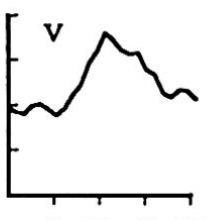

$\begin{array}{lllll}6 & 12 & 18 & 24 & \mathrm{Uhr}\end{array}$
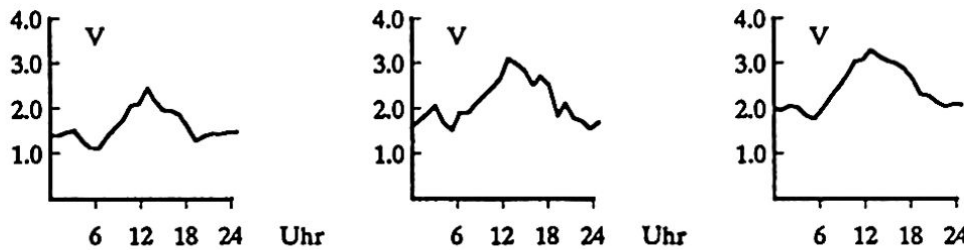

$\begin{array}{lllll}6 & 12 & 18 & 24 & \mathrm{Uhr}\end{array}$

Mittlere Windgeschwindigkeiten (in $\mathrm{m} / \mathrm{s}$ ) je Richtungssektor (1-36)
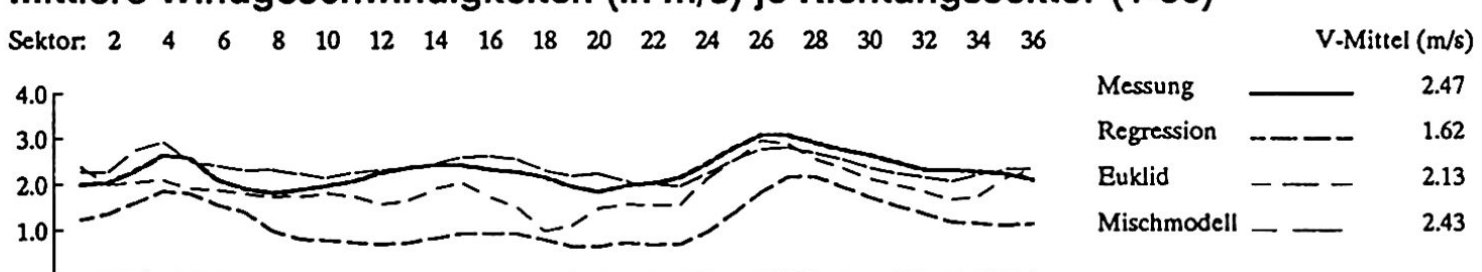

$\begin{array}{ll}\text { Vergleichsstatistik } & \text { Messung } \\ \text { Geschwindigkeit:Vektormittel (m/s): } & 0.96 \\ \text { Richtung:Vektormittel (Grad): } & 286 \\ \text { Vektor-Standardabweichung } & 2.64 \\ \text { Vektor-Korrelationskoeffizient: } & 1.00 \\ \text { Korrelation Windgeschwindigkeiten: } & \\ \text { Korrelation N- und E-Komponenten: }\end{array}$

Regression
1.09
284
1.72
0.68
0.70
N:0.59 E:0.71

Euklid
1.01
276
2.40
0.49
0.58

Mischmodell
0.95
275
2.47
0.47
0.76
N:0.33 $\quad$ : 0.55

Fuer Modelle verwendete Anetz-Stationen: Wynau,Aarau,Luzern,Waedenswil,Altdorf,Engelberg plus Temperaturdifferenz Luzern-Napf

Fig.5 Vergleich von Messung und zeitlich extrapolierten Winddaten. Angewendet wurde das Mischmodell (Auswertung B. RICKLI). 
den. Bei der Festlegung des Ähnlichkeitskriteriums sind Höhenstationen weniger stark gewichtet worden als Talstationen. Die Windgeschwindigkeit wurde mit Hilfe von Regressionsmodellen berechnet. Die Qualität der Schätzung der fehlenden Werte ist dabei abhängig von der Jahreszeit der Messungen, von der topographischen Lage der Station und auch von der Ähnlichkeit und der Nähe der permanent messenden Stationen. Als ein Beispiel für die Qualität der Schätzungen sei auf die Figur 5 verwiesen, wo für eine der permanent messenden Stationen, ausgehend von einer vierteljährlichen Meßreihe, für einen Zeitraum von neun Monaten die generierten mit den gemessenen Werten verglichen sind. Getestet wurden total drei Extrapolationsmodelle. Zur Anwendung gelangte schließlich das Mischmodell (Clusteranalyse kombiniert mit Regressionsanalyse).

Mit den eigenen sowie mit von Dritten bezogenen Meßdaten konnte eine Datenbank mit Jahreswinddaten von über 60 Stationen aufgebaut werden, wovon etwa 55 gleichzeitig während des Referenzjahres 1986/87 vorlagen.

Mit Hilfe dieser Daten wurden dann einerseits Ausbreitungsstatistiken berechnet, anderseits wiederum via Clustermethoden 18 typische Strömungslagen für den Raum Innerschweiz abgegrenzt und dargestellt. Diese Strömungslagen repräsentieren das Windverhalten aber nur auf dem 10-m-Niveau, zudem gibt es immer noch größere Gebiete ohne eigene Messungen, so daß eine flächendekkende Aussage noch nicht möglich ist.

In vielen Alpentälern läßt sich die Windrichtung einer einzelnen Station relativ leicht auf das übrige Tal übertragen, die Hauptwindachse liegt in den meisten Fällen talachsenparallel. Die räumliche Extra- oder Interpolation der Windgeschwindigkeit ist demgegenüber von weiteren Faktoren abhängig, aber auch hier können notfalls die Werte benachbarter Stationen übernommen werden, ohne daß große Fehler gemacht werden.

Im Mittelland ist das dynamische Verhalten des Windfeldes schwerer zu interpretieren. Deshalb ist hier der Versuch unternommen worden, das mittlere Windverhalten der bodennahen Luftschicht (Mächtigkeit: $50 \mathrm{~m}$, sogenannte Prandtl-Schicht) mit dem hydrostatischen, numerischen Modell FOOT (BORN, 1989) zu generieren. FOOT ist ein einfaches, prognostisches Dreischicht-Modell, das nach Eingabe von Topographie-, Rauhigkeits-, Temperatur- und Druckdaten sowie des geostrophischen Antriebs Windfelder in einer maximalen Auflösung von $2 \mathrm{~km}^{2}$ berechnen kann.

Eines der Hauptprobleme von FOOT ist dessen Anwendungsbeschränkung auf relativ ebene Gebiete: Geländeneigungen von über $10 \%$ können nicht verarbeitet werden. Damit können topographische Daten der Alpen oder des Juras nicht berücksichtigt werden. Das Modell kann demnach weder die durch die beiden Gebirge verursachte Kanalisierung im Mittelland noch die durch die Massenerhebungen der beiden Gebirge induzierten großräumigen ana- oder katabatischen Winde modellieren.
Das erste Problem kann durch Anpassung des antreibenden "geostrophischen» Windes angegangen werden, das zweite ist kaum zu bewältigen, da beispielsweise die Schiefstellung des Geländes (mit einem starken Anstieg gegen die Alpen zu) die übrige Modellrealität zu stark beeinflußt. Weiter fällt auf, daß die starke topographische Beeinflussung der Winde auf dem 10-m-Niveau in der Prandtl-Schicht deutlich weniger ausgeprägt ausfällt.

Für eine weiterführende Diskussion der mit FOOT erzeugten Resultate sei auf RICKLI hingewiesen (RICKLI, 1991).

\subsection{Resultate}

Die 18 erwähnten typisierten Strömungslagen können zur räumlichen Extrapolation von Ausbreitungsstatistiken (10-m-Niveau) herangezogen werden.

Etwa in $60 \%$ aller Fälle treten lokal-regionale "Talaboder Talaufwind-Strömungslagen» auf. Entlang des Jurasüdfußes ist dieser Prozentsatz geringer, in den Alpentälern größer. In der übrigen Zeit sind die Winde deutlich von der Höhenströmung beeinflußt.

Etwa $15 \%$ aller Winde weisen das lufthygienisch problematische «Talabwind»-Strömungsverhalten auf. Sie treten vor allem während der Nacht auf, während des Winters aber auch am Tag. Abfließende Kaltluft (Hangabwinde, Bergwinde) weist ein komplexes (intermittierendes?) Turbulenzverhalten auf, ihre Geschwindigkeit ist zudem gering: Schadstoffe werden demnach also hauptsächlich talachsen- oder geländeparallel und kaum vertikal transportiert, was zusätzlich durch darüberliegende wärmere Luft (Inversion) verhindert wird. Die lokalen Abwinde sind von den Höhenwinden praktisch ganz entkoppelt. Die mittleren Windgeschwindigkeiten bleiben im tieferen Mittelland meistens unter $1 \mathrm{~m} / \mathrm{s}$.

Knapp 20\% der Windströmungen entsprechen der Strömungslage "Talaufwinde» (Talwinde oder Hangaufwinde). Sie ist besonders während den Sommermonaten häufig anzutreffen, da vor allem bei starker Einstrahlung der Boden die darüber liegende Luft erwärmen kann, die dann relativ turbulent dem Gelände folgend aufsteigt. Höhenluft kann bereits leichter eingemischt und die bodennahe Strömung dadurch beeinflußt werden, die Windgeschwindigkeiten sind dementsprechend deutlich höher als bei der Talabwind-Lage. Auf- und Abwinde lösen sich in einem täglichen Rhythmus ab.

Die übrigen Strömungsmuster werden bereits weitgehend vom geostrophischen Wind beeinflußt, der über dem Mittelland durch Alpen und Jura und in den Tälern allerdings stark kanalisiert ist (Westwindlage, Bisenlage, Föhn). Die Windgeschwindigkeiten sind noch einmal höher als bei den Aufwind-Mustern, die Windrichtungen entsprechen vor allem bei starken Höhenwinden und eher instabiler Schichtung derjenigen des geostrophischen Windes.

Die Windrichtungshäufigkeiten in der Innerschweiz sind in der Windkarte (Fig. 6) dargestellt.

Die Windgeschwindigkeiten erreichen im Jahresmittel bei den Stationen unterhalb 1200 m ü.M. nur Werte zwi- 


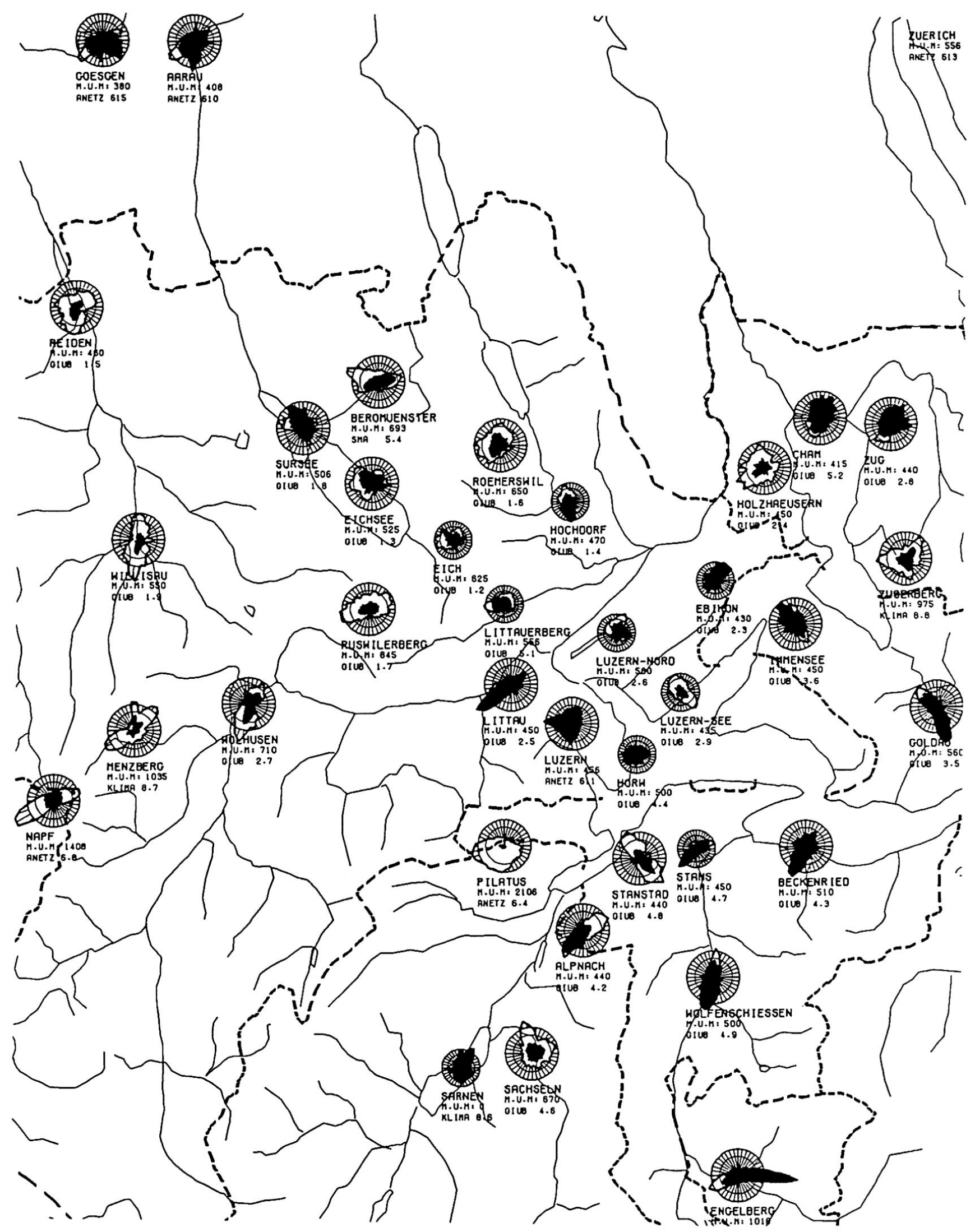

Fig.6 Windrichtungshäufigkeiten auf dem 10-m-Niveau für das Referenzjahr 1986/87: Modifizierter Ausschnitt aus der mehrfarbigen Windkarte der Innerschweiz. Werte gemessen oder zeitlich extrapoliert (Auswertung: B. RICKLI). Flächentreue Darstellung: Skala: 2,5 und $5 \%$ Häufigkeit.

Schwarz: Windgeschwindigkeiten «=1,3 m/s, weiß» $1,3 \mathrm{~m} / \mathrm{s}$. 


\section{Windhäufigkeiten im Kt. Luzern, Nacht-Stunden}

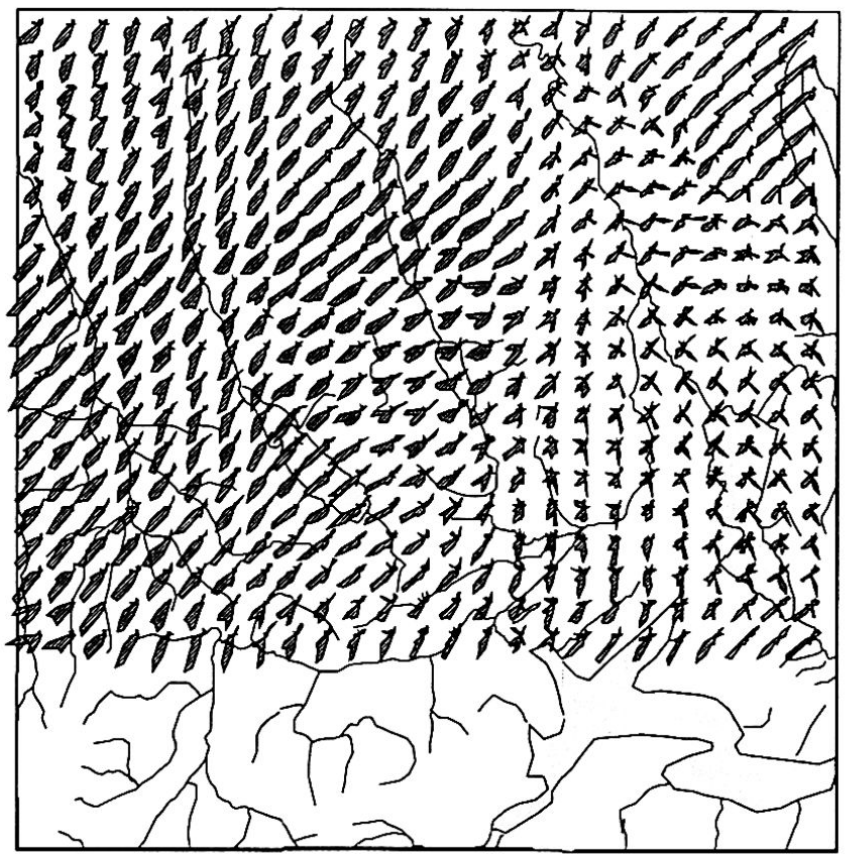

Geografisches Institut der Universitāt Bern, B.Rickli, 1990

Fig.7 Mittlere Windrichtungshäufigkeiten in der PrandtlSchicht für die Nachtstunden des Referenzjahres 1986/87, erzeugt mit dem Computermodell FOOT (Auswertung: B. RICKLI).

schen 1,1 und maximal $2,5 \mathrm{~m} / \mathrm{s}$, was als relativ niedrig angesehen werden muß. Derartige Werte sind aber durchaus typisch für die Durchlüftungsverhältnisse im schweizerischen Mittelland. Sie sind unter anderem dafür verantwortlich, daß auch kleinere Schweizer Städte mit beträchtlichen lufthygienischen Problemen zu kämpfen haben.

Die mit FOOT generierten Windrichtungshäufigkeiten für das Referenzjahr und die Nachtstunden zwischen 18.00 und 06.00 Uhr sind in Figur 7 dargestellt. Die Windrosen stellen die Windrichtungshäufigkeiten in linearer Form dar, d.h., die großen Häufigkeiten werden stark übertrieben dargestellt. Die seltenen kommen kaum zum Ausdruck, weshalb vor allem die nordöstlichen Richtungen als untervertreten erscheinen. Es wird deutlich, daß die Windrichtungen im westlichen Teil der Karte nur wenig in Richtung der von Norden nach Süden verlaufenden Täler (Suhretal, Wiggertal) kanalisiert werden. Das Klein-Relief kann dort vom Modell nicht in genügend großer Auflösung berücksichtigt werden. Erst über dem breiteren Reußtal im Kanton Aargau wird eine deutliche Kanalisierung beziehungsweise ein deutliches Abfließen von Kaltluft sichtbar. Die Hauptwindrichtung $\mathrm{SW}$ ist zudem etwas zu stark gegen S gedreht, was bei der Eingabe des Antriebs noch stärker zu berücksichtigen wäre (die Linksdrehung des geostrophischen Windes gegen die Prandtl-Schicht wird durch das Modell übertrieben).

\section{Ausbreitungsklassen}

Mit Hilfe der bisher beschriebenen Auswertungen konnten pro Windmeßstation auch sogenannte Ausbreitungsstatistiken berechnet werden, die direkt von Computermodellen wie den IMMPROG-Modellen (AIRINFO, 1990) zur Prognose der Immissions-Situation eingesetzt werden können. Es werden meistens sechs Ausbreitungsklassen von sehr labil (gute Verdünnung, hohe Turbulenz) über neutral bis sehr stabil (schlechte Verdünnung) ausgeschieden, die den Gesamtzustand der Atmosphäre bezüglich des Ausbreitungsverhaltens charakterisieren, also sowohl die thermische als auch die mechanische Turbulenz (via Windgeschwindigkeit) berücksichtigen (Fig. 8).

Der Raum Innerschweiz fällt durch einen hohen Anteil an stabilen Ausbreitungsklassen auf, dies besonders ausgeprägt in den Gebieten mit häufigen Kaltluftansammlungen. Stabile Bedingungen treten vor allem während der Nacht auf. Stationen in reliefungeschützten Gebieten (Höhenstationen) weisen demgegenüber vermehrt neutrale Bedingungen auf.
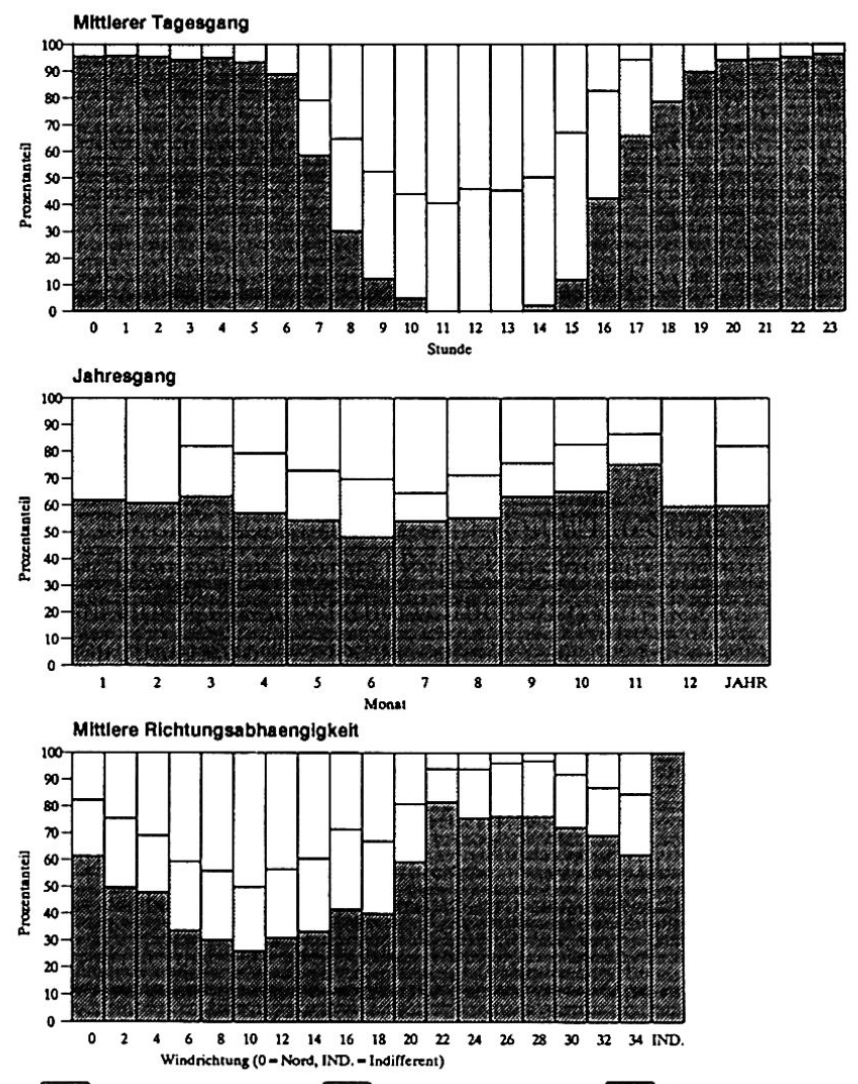

Lobil (Klassen 1 und 2) $\square$ Neutral (Klassen 3 und 4)

Stabill (Klassen 5 und 6)

Fig.8 Tagesgang, Jahresgang und Richtungsabhängigkeit der Ausbreitungsklassen der SMA-Station Luzern (Auswertung: R. PFEIFER). 


\section{Schlußbemerkungen}

Mit der vorgestellten Studie liegt erstmals für ein größeres Gebiet der Schweiz eine flächendeckende Charakterisierung des Ausbreitungsklimas vor. Die vorhandenen Auswertungen sind praxisorientiert und richten sich an den Lufthygieniker oder Ingenieur, der z. B. im Rahmen einer Umweltverträglichkeitsprüfung die Immissionsmehrbelastung abschätzen muß, die durch eine neue Quelle verursacht wird.

Aus klimatologischer Sicht ist wiederum die starke Beeinflussung des Ausbreitungsklimas durch die Topographie zu erwähnen: So weist praktisch jedes Gebiet sein eigenes Ausbreitungsverhalten auf. Kaltluft fließt hangoder talabwärts, sammelt sich in Muldenlagen oder vor Engnissen, läßt die anfänglich bodennahe Inversionsbasis ansteigen, und die Ausstrahlung verstärkt die Vertikalstabiliät weiter. Gestaute Kaltluft weist nur noch eine geringe Windgeschwindigkeit auf, die Windrichtung ist durch die Topographie gegeben, die Höhenwinde sind entkoppelt. Das Beispiel mag zeigen, wie stark alle am Ausbreitungsvorgang beteiligten Komponenten miteinander gekoppelt sind. Die Kaltluftgebietskarte und die Windkarte können deshalb zusammen mit den Ausbreitungsstatistiken als eigentliche Synthese der Studie aufgefaßt werden.

Aus wissenschaftlicher Sicht wäre die Beantwortung weiterer Fragen zu begrüßen. So wäre beispielsweise die Windfeldmodellierung mit höher auflösenden, nichthydrostatischen Modellen wünschenswert, da das angewendete Modell FOOT die räumliche Variation der Windverhältnisse des 10-m-Niveaus nicht erfassen kann. Interessant wäre sicherlich auch eine Studie über die Kopplungsmechanismen zwischen bodennahen Winden und jenen in der Höhe oder die Abschätzung interregionaler Transportmechanismen. Auch die Identifika- tion von Kaltluftgebieten kann in Zukunft mit Satellitenbildern höherer Auflösung durchgeführt werden.

\section{Verdankungen}

Das Projekt wurde von den Kantonen Luzern, Uri, Schwyz, Nidwalden, Obwalden und Zug finanziert. Mitgearbeitet am Projekt haben: F.X. Troxler, R. Pfeifer, T. Brunner, A. Estermann, E. Salvisberg, Dr. G. Nejedly, K. Flückiger, R. Kalbermatten, H. Gmünder. Das Geographische Institut der Universität Bern stellte einen wesentlichen Teil der Infrastruktur zur Verfügung. Daten lieferten die SMA, EAWAG, CKW und einzelne Kantone. Das Computermodell FOOT konnte vom Meteorologischen Institut der Universität Bonn übernommen werden, die Computeranlage stellte die Firma AirInfo AG in Bern-Liebefeld zur Verfügung. Frau Susi Schriber bearbeitete Text und Figuren. Allen beteiligten Personen und Institutionen sei an dieser Stelle herzlich gedankt.

\section{Literatur}

AIR INFO AG (1990/91): Immprog-P, Immprog-H, ImmprogC: Immissionsprognosen für Punkt- und Linienquellen: Benutzer-Handbuch. Airlnfo AG, Liebefeld.

BORN, K. (1989): Foot: Ein Dreischichtmodell auf der Basis von Rewimet zur Simulation der dynamischen Grenzschicht Met. Inst. Univ. Bonn.

RICKLI, B., P. FILLIGER, F.X. TROXLER, R. PFEIFER, T. BRUNNER, A. ESTERMANN, E. SALVISBERG (1989): Das Ausbreitungsklima der Innerschweiz. Geogr. Inst. Univ. Bern. 2 Bände.

RICKLI B. (1991): Windverhältnisse der Innerschweiz. Diss. Geogr. Inst. Univ. Bern. In Vorbereitung. 